\title{
Anchoring policy development around stable points: an approach to regulating the co-evolving ICT ecosystem
}

\section{Abstract}

The daunting pace of innovation in the information and communications technology (ICT) landscape, of technology and business structure, is a well-known but under-appreciated reality. In contrast, the rate of policy and regulatory innovation is much slower, partly due to its inherently more deliberative character. We describe this disparity in terms of the natural rates of change in different parts of the ecosystem, and examine why it has impeded attempts to impose effective regulation on the telecommunications industry. We explain why a recent movement to reduce this disparity by increasing the pace of regulation - adaptive regulation faces five obstacles that may hinder its feasibility in the ICT ecosystem. As a means to achieve more sustainable regulatory frameworks for ICT industries, we introduce an approach based on finding stable points in the system architecture. We explore the origin and role of these stable points in a rapidly evolving system, and argue that they can provide a means to support development of policies, including adaptive regulation approaches, that are more likely to survive the rapid pace of evolution in technology.

Keywords: Co-evolution, Internet regulation, standardization, architectural constraints, adaptive regulation

\section{Introduction}

Co-evolution, as defined in biology, is the dynamic process wherein two or more closely related species each change in response to changes in the other: flowers and the insects that pollinate them, or predator and prey. This term reflects the fact that all evolution occurs in an ecosystem, where the self-directed behavior of each actor (or species) may strongly influence, or sometimes even determine, the evolution of others. Competitive processes are also a form of evolution, where the "survival of the fittest" principle applies. In business, military, or government affairs, successful evolution requires the ability to drive advances in technology, and to incorporate new technical innovations into practice. This requirement to adapt is not unique or specific to information and communications technology; what is distinctive is the pace at which this technology advances, especially relative to the pace of development of business models and public policy. In this paper we explore the natural rate of change of various components of the ICT ecosystem, where some interdependent actors have a natural tendency to evolve faster than others. We will use the Internet as an example of such a co-evolutionary system, and consider the implications of the divergent rates of change for developing sustainable policy. We explore the concept of adaptive regulation, an emerging movement to render 
regulation more responsive to its rapidly change context. The promise of adaptive regulation faces at least five challenges that are particularly relevant in the ICT context: agreeing on policy goals, measuring progress toward them in order to inform the adaptation process, designing specific regulatory approaches that are fit for the specific purpose of the regulation, determining that a policy change did indeed cause the measured outcome, and dealing with potential harm induced from too rapid adaptation. Our engineering perspective lends skepticism to the belief that adaptive regulation will be a panacea for the ICT ecosystem. As a complement, we propose an approach to regulation based on finding stable points in the system around which to anchor public policies.

\section{Different rates of change across co-evolving ecosystems}

\subsection{The rapid pace of information and communications technology evolution}

Moore's Law (Moore, 1965) predicts the future rate of improvement in information technology. The prediction is actually an assertion about the best rate of investment in $R \& D$, not a law driven by physics. Moore's Law predicts that the best rate of investment will lead to the performance of information technology doubling about every 18 months, i.e., its growth is exponential. ${ }^{1} \mathrm{~A}$ doubling in 18 months grows by a factor of 10 every 5 years, 100 every 10 years, 1000 every 15 years. The speed of processors, the bandwidth of communication links (e.g., fibers), and the size of disks all grow exponentially. While the rates of growth may differ, the result is the same: technologies that engineers could only contemplate 10 or 15 years ago are now not only possible but practical at low cost, e.g., tablets, smart phones, streaming video, storing all of our data "in the cloud", real-time personal navigation systems. Even broadband was only an emerging aspiration fifteen years ago, when most Internet access was still dialup. Today we have consumer devices with amazing capabilities, some that often seem to outrun need. We not only have digital cameras, we have cameras that can recognize that a face is in a picture.

Moore's Law can be exploited by technologists in two ways: more performance for constant cost or constant performance for shrinking cost. For constant performance, costs may drop exponentially, e.g., by 10 every 5 years. Improving performance led to powerful new mobile devices such as tablets and smart phones, but continually shrinking costs have allowed companies to manufacture low-end smart phones overseas for less than fifty dollars, and essentially give them away in the developing world. ${ }^{2}$ Indeed, these forces have brought the developing world online.

Exact predictions about the trajectory and role of information technology may vary, but we will continue to experience its increasing penetration into every aspect of our lives. As more aspects of society go online, from money to war to political and

${ }^{1}$ Different versions of Moore's Law give 18 or 24 months as the period of doubling.

${ }^{2}$ Costs for such devices will not continue to decline indefinitely; some components like housings and batteries do not follow an exponential improvement curve. 
civic discourse, we will become completely dependent on this technology, and thus increasingly threatened by its complexity, opacity, and possibility of failure. Economic forces amid growing demand for information and communications technology can lead to instability and vulnerabilities, pressures that will trigger increasing calls for intervention and regulation by governments. ${ }^{3}$

\subsection{The different paces of technology integration across the ecosystem}

The rapid pace of Moore's Law drives rapid innovation in the private sector, lending advantage to those who invent, discover, or adapt to new technologies sooner than others. But as technology is integrated into industry and society, different parts of the ecosystem exhibit different dynamics, subjecting each part of the ecosystem to evolutionary constraints. The Internet offers an illustrative example of this highly heterogeneous industry structure. Figure 1 shows a variant of the well-known layered model of the Internet that allows us to describe some of this heterogeneity.

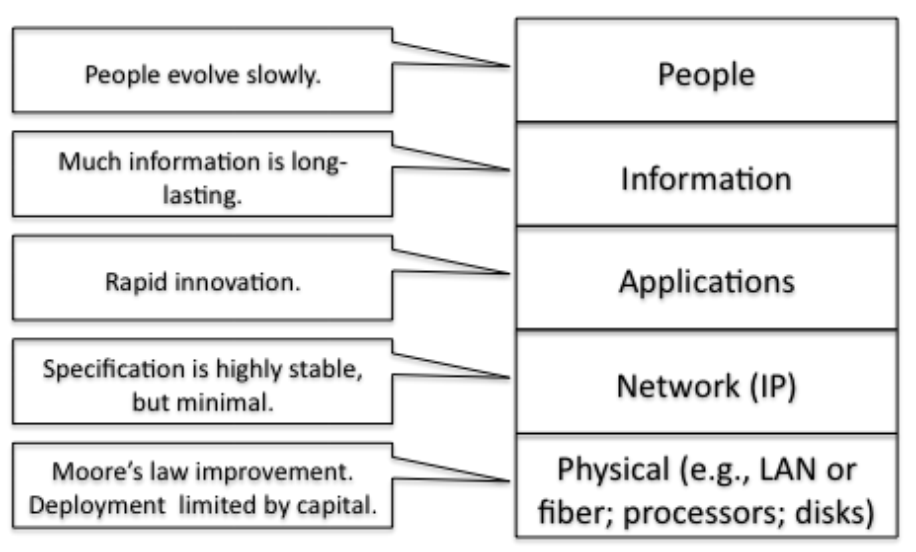

Figure 1: A layered model of the Internet ecosystem, describing the key dynamics of each layer.
The physical (lowest) layer experiences a rate of change gated by labor and sources of capital, neither of which follow a Moore's Law cost function. For example, the on-going massive rebuilding of the world's communications infrastructure, replacing old copper telephony infrastructure with fiber and radio, requires massive investment, so rates of return on new capital investment limit the pace of this evolution. Factors differ by region, but for many parts of the world, in 10 or 20 years, we will have largely completed this conversion to fiber optics. The resulting massive capacity will pay returns for several decades.

Cloud computing is another example of the interplay of Moore's law and capital investment. The large data center infrastructures supporting cloud computing benefit from both rapidly advancing technology and ever increasing massive arrays of computers. The limit to the capacity of a data center is not primarily Moore's law, but construction and operational costs.

${ }^{3}$ A vivid example of this sociological dynamic is ICANN's controversial plan to expand the DNS root zone by orders of magnitude, adding thousands of new top level domains (TLDs) with little understanding of its implications for the security and stability of the Internet (k claffy, 2011). 
At the Internet layer (the Internet Protocol, or IP), the durability of the specifications of the core protocols provides a stable foundation for rapid innovation at other layers. This stability was intentional (D. Clark, 1988), although even its designers did not appreciate just how difficult it would be to evolve the standard past this stable point. Such specifications do evolve, as we see with the current effort to convert the protocol to a new version that supports more addresses (IPv6), but at the pace of human debate and disagreement and highly decentralized investment decisions. ${ }^{4}$ The complexity of designing a system to accommodate changes to its stable points highlights an ossification view of architectural evolution, first applied to operating systems many years ago (Belady \& Lehman, 1976): a system undergoes continuing change until it becomes too brittle to accommodate effective evolution, and eventually it is more cost-effective to freeze and recreate a new system. ${ }^{5}$

At the application layer, the process of innovation is driven at almost frantic rates by the potential of a $10 \mathrm{X}$ improvement in underlying technology every 5 years. Innovators dream, wait, and move when the technology permits, to try out ever new and surprising concepts.

At the information layer, the creation, storage, search and retrieval of essentially all forms of data - information, content, knowledge - is moving on line. The explosion of information is relentless, not only due to exponentially dropping storage costs, but also because of the increasing recognition of the value of metadata. Meta-data is information describing other information, such as format, provenance, ownership, veracity of data, or abstracts and alternative representations of data, such as the identity of every person in a picture. We now record all transactions on line: every purchase, every query, every interaction. We bemoan the loss of privacy, which will be a pivotal debate in society in the next decade. But the data is being recorded, and it is hard to imagine this trend will be reversed. This trend calls for durability in data representation (e.g., formats), lest yesterday's data be unreadable tomorrow.

At the people level, we experience a transformative empowerment from the deployment of technology in the hands of humans. But human capabilities in no way grow on a Moore's Law curve. We do not get twice as smart, or twice as capable of processing information, every 18 months. So we drown in information overload, and

${ }^{4}$ The attempt to develop and deploy IPv6 is now in its third decade (D. Clark, Chapin, Cerf, Braden, \& Hobby, 1991) with limited success. The highly contentious standardization process yielded the decision to make IPv6 not backward-compatible with IPv4, which has arguably been the greatest obstacle to migration.

5 The Internet architecture itself exhibits aspects of this brittleness, an inability to accommodate new technological trends and societal demands, ehich motivated the U.S. National Science Foundation to fund researchers to consider "clean-slate" designs of the Internet architecture. This NSF program has an interdisciplinary scope, recognizing the coevolution of economic and legal systems with technological systems, and the value of considering non-technical requirements in the design process. http://www.nets-fia.net. 
call for even more technology to control the flood, which makes us even more dependent on the technology.

These different paces of technology integration across the ecosystem also influence the stability and agility of firms. Companies that have invested in physical assets like fibers to the home, towers or data centers can sometimes earn a stable place in the ecosystem through that investment ${ }^{6}$, although a bad technology bet can leave them disadvantaged by a stranded investment. Firms with extensive physical infrastructure investments also cannot easily move, and typically remain domestic except by merger and acquisition of firms in other countries.

In contrast, firms at higher layers are more likely based on an idea (like Facebook) than on heavy capital investment. The global uniformity of the packet forwarding layer of the Internet (the specifications that make it stable) means that an application running on top of this layer can be invoked anywhere, so firms providing services at the application layer may seek more favorable regulatory jurisdictions. The same international mobility may apply to the information layer, but both technical and jurisdictional issues may limit the scope of firms at the information layer.

The commercial ecosystem experiences constant pressure from application innovators to seek new capabilities from the physical layer (e.g., more capacity to the home), while the investment in those capabilities must be made by a different set of firms. This tension is a classic example of co-dependency and co-evolution within the industrial part of the ecosystem, where applications are limited in their ability to evolve by the rate at which the physical layer can evolve. Because the application layer depends on the physical layer, the application layer cannot simply out-evolve the physical layer, but is gated by it.

\subsection{The slower pace of law and regulation}

In contrast to the rate of change in the technology and industry structure of the ICT sector, law and regulation evolve more slowly, due to the need to resolve tensions among divergent objectives of members of the private sector(s) and the state(s). ICT firms at all layers are driven by economic motivations - pursuit of profits or economic survival, growth, or competitive advantage. These goals involve a uniform challenge: create products or services that customers and complementors prefer. In contrast, states have a range of objectives, sometimes unrelated, sometimes in tension with each other, and variable across nations, e.g., national security, internal order and public safety, taxation, economic growth and prosperity, and consumer protection. Many tools of the state - law and regulation, funding of research, direct expenditure of funds, leadership and convening, international negotiation and diplomacy - can serve a variety of purposes. But the pace of law and

\footnotetext{
${ }^{6}$ Network infrastructure companies may also engage in the regulatory process to preserve their dominance.
} 
regulation is gated by the need to deliberate to resolve tensions among objectives: control, accountability, investment, regulation, etc. This pace is unlikely to ever match the pace of the technology sector, by any measure.

The U.S. Telecommunications Act illustrates this pace of law. The Act of 1934 stood for almost 62 years until revised as the Telecommunications Act of 1996. That law is less than 20 years old, and in that time we have seen the explosion of broadband access, the rise and fall of the CLECs, the death of long-distance telephony as a competitive consumer-facing market, the rise of the cable providers as a facilities-based competitor in broadband access, and the possible emergence of cable as a dominant survivor in this competition. Many sections of that act seem dated now, but the prospect of rewriting it yet again is also daunting, especially given the continued rapid evolution of many ecosystems a re-write would affect.

Scheuerman (Scheuerman, 2004) has argued that the social changes brought on by the acceleration of technology development are a threat to legislative processes, and indeed to sustainability of the rule of law itself, and thus to market capitalism and liberal democracy. His concern is that the doctrine of separation of powers presupposes a different pace of dialogue within each branch of government - a slow, future-oriented legislature; a reactive, present-oriented executive; and a reflective, past-oriented judiciary - and that modern high-speed social engagement induces a conflation of these traditionally meaningfully disjoint tempos, and associated challenges for sustaining independence among the branches. He offers some suggestions for how each branch can cope with the acceleration, such as technology to facilitate more rapid public deliberation on policy questions, but most of his suggestions presuppose, without evidence, an electorate who wants to spend their free time following, much less engaging in, political debates. Although the impedance mismatch among technology, business dynamics, and policy is wellrecognized in the literature, it resists an effective remedy, or even ability to quantify whether the disparity is increasing.

\subsection{Four possible scenarios resulting from disparity in rates of change}

The Red Queen's hypothesis (VanValen, 1973) ${ }^{7}$ is an evolutionary hypothesis that an organism must constantly adapt not only to gain reproductive advantage but even to maintain fitness relative to systems with which it co-evolves, e.g., a parasite to its host (or vice-versa). Specifically, an adaptation by a population of one species may change the selection pressure on a population of another species, resulting in antagonistic co-evolution of species. This hypothesis is applicable to the dynamics of technology and regulation, where the accelerated pace of technology and industry dynamics relative to government and civic processes allows only a few possible resulting scenarios (or some hybrid of these scenarios).

${ }^{7}$ The Red Queen's Race is an event in Lewis Carroll's Through the Looking Glass where a chess queen and Alice constantly run but remain in the same spot. Van Valen honored Carroll's imagery in hypothesizing this corollary to his proposed "Law of Extinction" (VanValen, 1973). 
- Industry constantly out-evolves the controls of the state, rendering these controls meaningless or counterproductive-in the language of the hypothesis, not fit for purpose. This outcome will be less tolerable as government actors perceive increasing needs to intervene and regulate.

- The pace of evolution (industry innovation) slows, perhaps by explicit government intervention, to the rate at which the state can evolve its laws and regulation. Governments have resisted this option because they recognize the ICT sector as a powerful engine of economic growth. Note that if one sector is dependent on another, as with the dependency of some Internet applications on upgrades to the physical layer such as broadband access, the pace of evolution will slow to the rate of the slower actor.

- The pace of evolution in regulation speeds up to match the pace of technical evolution. In the next section we explore one movement in this direction adaptive regulation - and its challenges. In general, this scenario requires more nimble processes for developing policy than we have today. This more dynamic approach could also lead to a less stable regulatory regime, adding uncertainty to the overall ecosystem and perhaps reducing the integrity of legislation by rushing into it (Cherry, 2008; Hubbard, Scott, \& Zingales, 2008).

- Regulators anchor policies around stable points in the system architecture, so that other components of the ecosystem can evolve at their natural pace.

In practice we may see hybrid blends of these outcomes, but as a tool for analysis and understanding, we examine the four options as distinct outcomes. We consider the first scenario, loss of fitness, unacceptable, so a successful approach must be some blend of the remaining three. The second scenario describes much of the history of wireless technology development, as spectrum policy limits what technologies can profitably deploy and thus influences investment decisions (Section 4.5). Although blocking technological innovation was not an explicit goal during the early history of spectrum policy, neither was promoting innovation; this history explains the resulting drag on innovation. Spectrum regulation arose in an era when the government defined acceptable uses of spectrum (e.g., broadcast radio or TV, or public safety), and specified technical standards. There was little expectation of rapid innovation, industry structure changed slowly, and the primary goal of regulation was the diffuse objective of "serving the public interest" as defined by the FCC. In this era, new ideas entered slowly. Coase first proposed spectrum auctions in 1959 (Coase, 1959); they were first utilized in 1994, 35 years later. Section 4.5 reviews how the last few decades have dramatically changed the context of spectrum regulation, where neither society nor industry is willing to accept the implications of technological evolution slowing to the pace of traditional policy evolution.

Thus, we consider the last two the most constructive alternatives. With respect to the third outcome, we discuss the limits to the rate at which regulation can adapt and evolve, from an engineering perspective. The fourth option relies on the 
presence of stable points in the design of technology and business structure. We believe that finding and exploiting such stable points of control is a promising approach to developing sustainable policy in the highly dynamic ICT ecosystem, and can likely improve the feasibility of adaptive regulation approaches.

\section{Increasing the pace of evolution in regulation}

\begin{tabular}{|l|l|}
\hline 1 & Agreeing on policy goals \\
\hline 2 & Measuring progress toward those goals \\
\hline 3 & Designing regulatory options intended to move toward those goals \\
\hline 4 & Determining that policy changes indeed caused observed outcomes \\
\hline 5 & Dealing with potential destabilization of ecosystem, due to rapid policy adjustments \\
\hline
\end{tabular}

Table 1: Five requirements for genuinely adaptive regulation (Section 3)

The term adaptive regulation has been used to describe the goal of regulation becoming more responsive to its rapidly changing context (Cherry \& Bauer, 2004; Cherry, 2007, 2008; Whitt, 2009; Longstaff, 2003; DeVries, 2011). Cherry and Bauer have used the telecommunications ecosystem to illustrate how policies are outputs of and inputs to co-evolving complex adaptive systems, along with technology and economic developments, and as such, policy analysis must "shift from its traditional paradigm of attempting to direct outcomes through optimization of parameters such as efficiency, to an evolutionary paradigm that emphasizes adaptability" (Cherry \& Bauer, 2004, page 13). Adaptation implies feedback, i.e., that the regulator can observe the evolving state of the system, monitor the effects of adopted policies, and experimentally adjust its regulatory stance to move the system toward desired goals (Cherry, 2007). At a high level, the idea of adaptive regulation seems compelling if not necessary to navigate today's ICT ecosystem dynamics. From an engineering perspective, the question is not whether the framing is valid but how to reduce it to practice. What are the operational issues in designing a regulatory regime that is adaptive- that is, one that can speed up the pace of evolution? We propose that for a regulatory scheme to effectively adapt, i.e., display auspicious coevolutionary behavior, it must meet five requirements: establishing measurable policy goals; measuring progress toward those goals; designing regulatory options intended to move toward those goals; determining that policy changes indeed caused observed outcomes; and dealing with potential destabilization of the overall ecosystem, due to rapid policy adjustments.

\subsection{Agreeing on policy goals}

Regulations can specify rules of behavior (inputs), or specify goals (outcomes). For example, building codes may mandate specific practices (e.g., "framing studs must be $2 x 6$ on 16 inch centers"), or they may specify outcomes, e.g., the building 
must remain standing under defined adverse circumstances. Both approaches have problems: outcomes are hard to specify crisply, and one can comply with rules in ways that cut corners and fail to achieve the intended outcome.

Specification of outcomes is likely to be more stable than specification of rules. The outcome that "buildings shall not fall down" has a timeless quality, but the rules and methods to achieve it (the inputs) may evolve rapidly. In an adaptive system, we would expect outcome specifications to change more slowly, and rule specifications to change more rapidly as part of the process of dynamic adaptation.

An intellectually principled regulation specifying rules would link those rules to the desired outcome (e.g., the objective of the regulation). Coupling of inputs to outputs in a regulation can allow for a range of regulatory options and increase flexibility for both regulators and practitioners. For example, in the case of building codes, one need not comply with default rules if an architect has certification from a licensed engineer that the design will meet the required outcomes. This allowance may complicate the job of a building inspector, but accommodates the reality that a single-family dwelling and a high-rise office building may achieve required outcomes using different designs. This flexibility provides firms freedom in meeting a given regulatory constraint: choosing either to undergo an ex post rule-based inspection or applying ex ante for a determination that some particular practice, while deviating from the rules, will achieve the desired outcome. Allowing this ex ante alternative might make it easier to specify tighter default rules.

An important factor is that the same rule may support multiple high-level goals. The case of network neutrality or "reasonable network management" illustrates a rule-based regulation that promotes potentially divergent objectives, e.g., innovation (Barbara van Schewick, 2010) or the production of downstream public and social goods (Frischmann, 2012). The world is messy and contentious, with competing aspirations and priorities, and advocates for different high-level outcomes may rally around the same rule for different reasons. This diversity limits the ability of the regulator to adapt the rule, as success may be measured against different objectives by different advocates.

We acknowledge, but do not review here, the wide range of mechanisms that contribute to the regulatory process. In particular, higher-level policy goals may be captured in law, rather than as a component of regulation; in fact one could argue that regulatory agencies should not be setting policy, only enforcing it. In this respect, we consider the regulatory process and its larger context as somewhat of a "black box", for further exploration by experts. Our point here is simple: one needs a clear articulation of a policy goal in order to effectively pursue it.

\subsection{Measuring progress toward policy goals}

The second challenge of taking such an experimental or adaptive approach to regulation is achieving agreement on how to monitor the effects of adopted policies. The process of adaptation requires measurement of progress with respect to the outcomes, not merely compliance with the rules. The above building code example illustrates a situation where both rules and goal evolve slowly. There is a general 
understanding that a single family home with studs every 16 inches will not fall down, so the building inspector can limit his attention to compliance with the rules, not the goals. When this linkage is less clear, an adaptive regulation must include a plan for how to measure progress with respect to the goals.

For this process to succeed, outcomes must be defined with clarity and precision amenable to measurement. Historically, such precise specification of policy goals has been elusive. Consider the proposed outcome: "the government must be able to intercept a voice call". Although at one time this outcome might have been a crisp specification, today it is quite unclear. What is a voice call? Should this order cover a Skype conversation over the Internet? What about an audio conversation in a multiplayer game? Or an application that criminals develop precisely to avoid intercept? Can some party be held responsible for blocking or intercepting that conversation? And how would one measure compliance, given that anyone can develop a voice application and run it between two machines on the Internet?

As another Internet-related example, the high-level goals of availability, affordability and reliability specify admirable general directions toward which regulation should strive (Cherry, 2008), but for these goals to serve as operational targets of adaptive regulation, we must further agree on how to define them in measurable terms. One must first parameterize them: availability of what, affordability of what, and reliability of what? In the days of telephony, there was only one service level: a 64Kbps connection to carry a voice call. Today, there are a variety of broadband services - so we must specify what availability means - access to the Internet at some minimum speed, in one or both directions? Are mobile and fixed services to be treated interchangeably for the purpose of defining availability? What about mobile voice vs. data services? Revising the definition of broadband, as the FCC has done (Federal Communications Commission, 2015), results in a change to the measure of availability, and reminds us that goal-setting is a political process. Stating goals in a durable form (e.g., "buildings shall not fall down") may be one key to effective regulation, since having to continually renegotiate the terms of the specified goal is not conducive to effective and timely evolution.

One attempt to define "broadband" in a long-term, durable way is in a National Academies report on deployment of broadband (Computer Science and Telecommunications Board, 2002). Recognizing the difficulty in quantifying a broadband availability goal given the rapid evolution of the ecosystem, they chose to define broadband in terms of a high-level objective that was durable over time:

- Broadband Definition 1: Local access link performance should not be the limiting factor in a user's capability for running today's applications.

- Broadband Definition 2: Broadband services should provide sufficient performance and wide enough penetration of services reaching that performance level to encourage the development of new applications.

This definition implies that the quantified objective would evolve over time, based on the performance of an evolving basket of applications. They believed this 
approach might provide a signal to all actors as to how the quantified target would advance.

Similarly, setting goals and measuring progress toward them may require more quantitative intermediate goals. For example, Whitt proposes as a high-level aspiration the production of "more good ideas", i.e., innovation (Whitt, 2009), a difficult metric to measure or target. He thus proposes specific intermediate goals: regulators should harness broadband networks as optimal on-ramps to the Internet, specifically rendering such networks: (1) available, (2) of sufficient capacity, and (3) accessible, i.e., open or neutral. He then describes dimensions of availability (ubiquity, mobility, and choice), acknowledging that these intermediate objectives may imply trade-offs, such as balancing affordability and ubiquity against the goal of commercial investment in higher capacity. This attempt to reduce a high-level aspiration to specific and measurable objectives is laudable but the assumptions underlying Whitt's analysis are not universally accepted. Society may not prioritize "more good ideas" above, for example, universal access. Nor is the logic obvious that the production of "more good ideas" is optimized by the specific intermediate goals he proposes for broadband. Finally, he prescribes a reasoned but not obvious balance among the dimensions of these outcomes. This sort of complexity is the essential and sometimes intractable context of adaptive regulation. And as with other engineered feedback systems, if there is any signal to detect as a basis for action, it may take a long time to extract it from surrounding noise. In the case of a socio-political system, this noise includes the clamor of competing goals and competing approaches to those goals, as well as the often overwhelming forces of special interests.

\subsection{Designing regulations that move system toward intended goals}

At a high level, one can state the necessary quality for a regulation to be sustainable: that it appropriately achieves its intended goals. Cherry and Bauer propose that "sustainable policies are defined as rules that are politically adoptable and for which the desired policy goals are reasonably likely to be achievable" (Cherry \& Bauer, 2004). This specification states a highly plausible requirement, but it is a requirements statement, and as such does not offer guidance as to how to achieve such policies. Rather than providing structured guidance in designing regulation, the co-evolutionary framing serves as a reminder that all regulatory proposals are experiments, and must be subject to revision and replacement if they prove unfit in practice.

\subsection{Determining that policy is actually causing measured outcomes}

Even if one can measure progress toward or away from a policy objective, that does not mean that the specific policies in place are causing that movement. The fourth challenge is thus determining causality: the ability to argue that the regulation caused an outcome, rather than merely exhibited correlation with it. Industry and governments can run experiments in parallel, bringing different ideas to market to see which work. The political concept of federalism implies a level of experimentation among local governments, acknowledging some limits imposed by 
federal laws. Cherry has discussed federalism as a patching algorithm, where the results of parallel regulatory experiments (different states or countries) can be compared and re-integrated into future experiments (Cherry, 2007). Running experiments in parallel, whether among states within a federal context or among different nation states, is a powerful way to learn more quickly from practice. However, potential path dependencies among governments may challenge comparative analysis, particularly when assessing causality. There may not be a sufficient number of regulatory regimes in the world to accommodate effective learning, given contextual differences across regimes. Parallel experiments can also be costly, since firms must navigate regulatory requirements across different regions.

Manzi (Manzi, 2012) offers a lively discussion of how social science can, and should, improve its practical utility by using a long-standing experimental approach, randomized field trials, to justify conclusions about causality. But he acknowledges that even with such improvement, social science will not be able to adjudicate most important policy debates. Drug trials, which may involve thousands of subjects and years to evaluate, illustrate the challenge of trying to justify regulatory adjustment in support of a high-level goal. A causally rich space warrants caution, which tends to imply an inherently slower pace of adaptation.

\subsection{Ecosystem instability due to rapid changes in policy}

Because regulation and law set rules for industry, we should not expect regulation to change as fast as the rest of the ecosystem. If rules change too fast, instability can result. In engineering terms, if a system adapts too quickly, the result can be oscillation and unstable runaway behavior. An adaptive approach will imply structural limits to the speed at which regulatory experiments can proceed.

\section{Anchoring policy development around architectural stable points}

Adaptive regulation is a useful concept, but its social and political viability for communications policy remain questionable (Ruhl, 2005); in practice it has proven far more difficult than its early advocates expected, and it likely requires substantial changes in administrative law to support its use in a given industry (Craig \& Ruhl, 2014). Our engineering perspective reinforces our doubts that the adaptive regulation framework alone can meet the challenges of today's ICT ecosystem, and lends inspiration to our exploration of the fourth alternative scenario of the Red Queen dynamic described in Section 2.4: finding stable points in a ecosystem to which to attach regulatory obligations, to allow both rapid evolution of other parts of the system while maintaining some regulatory control.

\subsection{The role of stable points in a rapidly evolving system}

Stable points are constraints within an ecosystem that free other parts of the ecosystem to evolve without (or with fewer) constraints. Stable points often 
manifest as interfaces between parts of the system. An interface is a set of standards or specifications that describe relationships among components: defining the functions each provides to others, and interdependencies each has on others. Stable interfaces change what might otherwise require coordinated innovation into independent innovation, on the separate sides of the interface. Because the interface is stable, modules on either side of it can evolve independently, so long as they conform to the specification. In 1998 Kirschner and Gerhart (Kirschner \& Gerhart, 1998) explored this concept in the context of evolution in the natural world. They concluded that since the ability to evolve productively is a key requirement to remain fit for purpose, and stability at key points in a system enables productive evolution, stable points may emerge as a part of natural selection as a consequence of the success of the resulting organisms.

In the ICT ecosystem, stable points sometimes emerge as a part of evolution, but they are often explicitly designed into a system architecture. Creation of stable points is an objective in designing long-lived systems, part of the art that good computer system designers practice. ${ }^{8}$ System designers specify critical interfaces as part of their system architecture, with the assumption that the interfaces (and their presumptive stability) will lead to its success. Different system architectures then compete in the marketplace. Systems that succeed proceed along a trajectory of increasing architectural stability (sometimes called lock-in.) The more parties that use an interface, the harder it is to undertake change in that interface. Systems that fail fade from view, along with their interfaces.

The importance of stable interfaces in the longevity of a system leads us to believe that regulatory objectives are more likely to be sustainable if they are attached to these stable points, such that the regulations can remain in force and fit for purpose even as the whole system evolves. Three examples of stable points in the ICT ecosystem make this approach more concrete:

- The basic Internet Protocol (v4) specification (Postel, 1981) has not changed since the beginning of the 1980's, providing a fixed anchor that allows technology below it (physical transmission technologies) to evolve independently from applications above it. So long as both the transmission technology and the application conform to the interface specification that links them, innovation is freed in both spaces.

- A processor's instruction set (the set of basic steps a computer can execute) is a somewhat stable point. Since processors (hardware) and software are usually not all replaced at once, a new processor must be able to run the same programs (e.g. execute the same instructions) as the old one. A move from one instruction set to another is a major transition, although not as major as replacing the specifications of the Internet.

- Information formats are a stable point. Because information is usually intended to be long-lasting, data formats (e.g., Portable Document Format

${ }^{8}$ For one discussion of alternative theories of longevity in network design, see (D. D. Clark, 2009). 
or PDF) are designed for longevity. If formats are replaced and/or software to process old formats is lost, information itself can become unreadable and effectively lost.

These three examples can also illustrate how stable points can limit or support mechanisms for regulation and control:

- If the original Internet Protocol (IP) specification had included a requirement for identity, we would have more accountability but no anonymity. The design choice was explicit, and one of us has argued (D. Clark \& Landau, 2011) that embedding accountability and identity into the specification of the Internet would be ineffective and contrary to important societal needs (at least from a U.S. perspective) such as anonymous speech and right of assembly.

- If processor instruction set specifications included mechanisms for protecting copyrighted material (e.g., Digital Rights Management or DRM hardware), the architecture of content control would become more durable. This idea is contentious: it may meet the needs of copyright holders but at too high economic and public costs. ${ }^{9}$

- If document formats such as PDF included, in an unforgeable way, the identity of the creator, one could verify the provenance and validity of a piece of information (assuming that the author elected to sign it) without having to confirm its source. This idea is not highly contentious, and its lack is probably a serious gap in the design of the Internet architecture.

Although stable interfaces can turn a tightly coupled system of co-evolution into a more loosely coupled system where actors evolve independently, interfaces seldom define all potential interactions among modules. For example, one of the few explicit design goals of the Internet architecture (i.e., protocol standards) was interoperation (D. Clark, 1988): to allow heterogeneous networks to connect to allow end-to-end packet carriage, and to allow heterogeneous computers to exchange those packets. The Internet Protocol specification says nothing about performance, interconnection methods, security, or reliability (Postel, 1981). The specification is only 31 pages, not including appendices. This minimality has benefits: it promotes product differentiation, since different providers of service can offer different levels of performance, reliability, security, etc. If the architects of IP had tried to write a more expansive specification, the whole process might have failed, since there was little understanding, let alone agreement, as to how to specify these other dimensions. Any stability in performance or security features has emerged organically as the system has evolved.

\subsection{The role of stable points in industry dynamics}

Stable points not only define interfaces between technical modules; they can define interfaces between firms, which can frame industry structure itself. In an argument that can be traced directly to Coase, boundaries between firms arise at

\footnotetext{
${ }^{9}$ For example, https://www.eff.org/issues/drm .
} 
points where transaction costs are low, and one way to reduce transaction costs is well-defined interfaces. Stable interfaces facilitate competition among companies providing comparable products. Regulators focusing on stable points are focusing on boundaries among firms, where regulatory attention often naturally arises.

With respect to industry structure, the specification of the Internet Protocol serves to insulate the evolution of technology below the interface from the evolution of applications it, but that interface does not decouple the dependence of applications on economic investment in infrastructure at the lower layer. With luck and good design sense, an interface may decouple the processes that have the highest rate of change - the technology itself - from the slower-paced interactions that may be the target of regulation. This outcome has occurred with some aspects of the Internet ecosystem. Debates about network neutrality are essentially attempts to impose additional constraints on the interface between the provider of the Internet layer and complementors on top of that layer.

The IP interface (Postel, 1981) is not the only aspect of the Internet ecosystem to emerge as a stable and constraining interface. Many different (potentially) stable interfaces exist in the Internet ecosystem, with different roots, trajectories, and lifetimes. The Border Gateway Protocol (BGP), which defines the traffic routing rules for interconnected ISPs, is a stable interface, deriving its stability both from its specification as a standard and from its deep embedding in industry practice. Many companies take explicit action to create and support an interface they hope will become stable, e.g., an interface used by third- party developers to implement applications on top of that platform. For example, Facebook, in its management of its third-party developer interface, first restricted itself to Harvard students, then only college students, then high school students, before opening the service to everyone. Facebook also tightened restrictions on its third-party application developers, from an initially open interface that imposed quality expectations via user ratings in their app store, to an explicit set of restrictions on developers (Boudreau \& Hagiu, 2011; Schilling, 2011). These crafted steps to embed their interface as a stable point led to accusations of platform mismanagement (McKenzie, 2013). 


\begin{tabular}{|l|l|l|}
\hline Release & Date & Standard \\
\hline & 1992 & 2G \\
\hline R98 & 1999 & EDGE, GPRS (2.5G) \\
\hline R99 & March 00 & UMTS (3G) \\
\hline R4 & March 01 & \\
\hline R5 & June 02 & IMS, HSPA \\
\hline R6 & March 05 & HSPA enhancements \\
\hline R7 & Dec 07 & HSPA+ \\
\hline R8 & Dec 08 & LTE (Pre 4G) \\
\hline R9 & Dec 09 & \\
\hline R10 & March 11 & LTE-E (4G) \\
\hline R11 & Sept 12 & IP interconnection \\
\hline R12 & June 14 & \\
\hline
\end{tabular}

Table 2: 3GPP release schedule of cellular standards, showing consistent somewhat predictable transitions between generations. Source: http://www.3gpp.org/

\subsection{The role of standards in the definition of stable interfaces}

Many stable interfaces, especially open interfaces used by third parties, are specified as standards. Standards are a path, perhaps the path, to creating points of control for regulators in cyberspace. Standards bodies are themselves embedded in the co-evolutionary ecosystem, and while their mode of operation is deliberation and consensus-building, like firms they are subject to pressure to evolve in order to remain fit for purpose and survive. State-centered organizations such as the ITU claim their legitimacy by virtue of international treaty, and thus by the power of the collective sovereign states. Bottom-up private sector standards organizations have no such claim; they survive only by defining standards that effectively meet the needs of industry, and firms will only send employees to participate in standards meetings likely to result in standards that help them evolve and compete. The traditional state-centered institutions like the ITU experience limited evolutionary pressure, but they embody the traditional pace of international deliberation, making them poorly structured to match the pace of cyberspace technology evolution, so 
they risk being out-evolved. ${ }^{10}$ These considerations suggest that a regulator or other actor interested in influencing a standard should carefully consider which standards-setting venue is likely to be most fit for purpose, in terms of speed of process and quality of outcome.

Not all standards are created with the goal of long-term durability. Some standards are planned with a known timeline of development, deployment, and obsolescence, providing an opportunity for active integration of regulatory concerns. A good example of such a standards process is $3 \mathrm{GPP}$ cellular standard development, a process that produces a release about every year, and a major revision (e.g., going from $2 \mathrm{G}$ to $3 \mathrm{G}$ to $4 \mathrm{G}$ ) about every 10 years (Table 2). This release schedule reveals four interesting aspects of standards evolution.

First, this release schedule provides consistent, industry-wide guidance to the rate of capital investment in new technology, similar to Moore's law. It aligns vendors and operators, reducing the cost of technology and preventing overenthusiastic and financially unjustified competition.

Second, most releases require more than a year to produce, so development of different releases overlaps. An analogous approach to policy, with groups working in parallel on different releases of a regulation, might accelerate progress but is almost unimaginable.

Third, standards bodies interact with each other at different rates of progress. For example, the ITU-R (the ITU radiocommunication sector) first provides a general specification of requirements for generations of cellular systems (in this case the Advanced Radio Interface Technology), but no guidelines for implementation. Then organizations like 3GPP develop detailed technical standards using their internal processes and ask the ITU-R to bless them as conformant. ${ }^{11}$ What results is an ITU standard developed by another standards body, using the processes of that other body. At the same time, the 3GPP also incorporates and builds on the Internet Protocol standard from the IETF. Standards bodies, like actors in other parts of the ecosystem, both compete and cooperate, a condition sometimes called "co-opetition".

Finally, governments are both directly and indirectly involved in the processes of the 3GPP. The U.S. organizational partner of 3GPP is ATIS (the Alliance for Telecommunications Industry Solutions), which includes as members the

10 The ITU has taken steps to speed up its standard-setting process in an attempt to be more relevant (ITU-T, 2001).

${ }^{11}$ Quoting from the 3GPP web site: "From 3GPP Release 10 onwards - 3GPP LTEAdvanced has been approved by ITU Radiocommunication Sector as a ITU-R IMTAdvanced Radio Interface Technology. The LTE standard now provides for peak speeds of $100 \mathrm{Mbit} / \mathrm{s}$ for high mobility and $1 \mathrm{Gbit} / \mathrm{s}$ for low mobility communication." Source: http://www.3gpp.org/About-3GPP, visited July 26, 2013. 
Department of Commerce and the Electronic Surveillance Technology Section of the FBI.

\subsection{Leveraging stable points for Internet regulation}

Literature on industry behavior tends to describe stable interfaces in terms of corporate control of platforms: a successful platform may give its creator and owner a powerful place in an ecosystem of firms (Gawer, 2011). In prior work (kc claffy \& Clark, 2014), we have used theories of multi-sided layered platforms (MSPs) (Hagiu \& Wright, 2011; Boudreau \& Hagiu, 2011) to explore several recent and impending innovations in the ICT ecosystem that have been conflated with the global Internet. Our goal in that work was to present a model of Internet industry practice general enough to survive current rates of innovation and evolution. We drew on two extremely stable features of today's telecommunications ecosystem: the use of layered platforms to implement functionality desired by either users or providers; and interconnection between actors at the same platform layer to construct a larger fabric.

We began this work by defining a taxonomy of platforms classified across two dimensions: how they are constructed (one or multiple firms) and how they are used (only by the platform owners or by others). The larger Internet ecosystem is many layers of platforms that span all four combinations of these two dimensions. The highly dynamic nature of the industry implies that technical details of platform layers will change faster than regulatory debate can resolve, but by focusing on stable points in the ecosystem, our taxonomy supports reasoning that is independent of technical details of the platform's construction or scope. We then analyzed two particularly stable platform layers of the ecosystem - the global Internet and the single-firm IP platform - and contrasted them with a third emerging platform innovation that raises significant challenges for regulators: the multi-firm IP platform. These layers represent degrees of freedom for regulators as well as industry actors.

We then constructed and used a layered multi-sided platform (MSP) model to explore several industry innovations that have been naively conflated with the global Internet, and described how regulators could use this model to more rigorously consider them. For example, we explored how a platform-aware model can distinguish among services running on different IP-based platforms, and the possible behavior of platform owners, in a way that offers a cleaner derivation and interpretation of their regulatory implications. For example, with respect to issues related to interconnection, the model makes a fundamental distinction between the role of multi-firm production and complementor interconnection. That is, peering among firms that make up the global Internet raises different issues than interconnection between a complementor and the Internet. To illustrate its potential as a baseline for future research, we showed how the model could help scope consistent policy discourse of three open questions: specialized services, minimum quality regulations ("the dirt road" problem), and structural separation. But the essential aspect of this model, and what we consider the source of its power, 
was its focus on the stable and persistent aspects of the existing Internet network and industry architecture.

\subsection{Stable points in the regulation of wireless communication}

We have used the Internet to illustrate the concept of stable points and dynamics in an ecosystem. We offer another example - the wireless industry - that provides additional insights about tradeoffs among stable points, rapid evolution and slow regulation. As described in Section 2.4, one outcome of the Red Queen dynamic is that innovation slows to the pace of regulation. We believe that the history of the wireless industry illustrates this outcome, and we will argue that the transition to a regulatory approach that stresses rapid innovation and adaptive regulation has led to the identification of new stable points in the architecture of wireless systems.

Spectrum regulation has been a regime of giving authorization: that which is not permitted is forbidden. In contrast, the Internet world has traditionally been the opposite: that which is not forbidden is permitted. Defining what is allowed rather than what is not allowed will tend to slow innovation to the rate of regulatory change. The now ubiquitous cellular voice and data transmission industries have gradually motivated a transition in thinking about spectrum policy over the last few decades, and the recent explosion of wireless ICT industries has put pressure on the rate of spectrum policy innovation. The recent history of spectrum regulation shows both a shortening of the regulatory cycles, and an increasingly flexible view of spectrum rights. As a data point, the first FCC docket on cellular services (No. 18262) opened in 1968 and led to an Order issued about ten years later. The duration of proceedings has shortened since then, although it may not qualify as adaptive. Reallocating spectrum can still take a decade if it requires moving current users out of spectrum bands they are using, prompting strong recommendations to abandon this strategy and move toward a model of sharing spectrum with existing incumbents (Federal Communications Commission, 2002; President's Council of Advisors on Science and Technology, 2012).

The 2002 FCC Spectrum Policy Task Force report (Federal Communications Commission, 2002) laid out a new direction and approach for spectrum policy, quoting FCC Chairman Powell stating, "the government has an almost impossible task in trying to keep pace with the ever-increasing demand for spectrum and the continuing advances in wireless technology and applications". The report called for flexible but clear rules for those granted rights, the elimination of barriers to use, and periodic review of rules (every 5-10 years, to balance needs for deliberation with needs for investment certainty). The report also explicitly called for a shift in the regulatory regime to a "that which is not forbidden is permitted" approach (Federal Communications Commission, 2002, pg.22), which requires clear rules that define interference among users, a durable constraint that also would de-constrain various actors, In other words, measurable interference provides a stable point around which to anchor a more adaptive policy: so long as spectrum users comply 
with interference requirements, they may use the spectrum as they please. ${ }^{12}$ This type of policy illustrates our main thesis, which is that the pace of regulation can align itself with fast-paced innovation by concentrating on stable interfaces or boundaries that decouple entities, so as to allow for innovation across the ecosystem. This policy also illustrates the value of regulating outcomes rather than inputs, and of aligning with user incentives. However, like many other spheres of regulation, there is not always agreement on the goals of spectrum regulation, which will inhibit effective adaptive regulation.

\subsection{Stable points for other regulatory goals}

We speculate about the potential role of stable points in achieving other goals for our telecommunication ecosystem. We note the distinction between stable points in a technological system and stable goals of a policy framework. A common manifestation of a stable point is an interface between two modules of a system, which often maps to an inter-firm boundary in the resulting ecosystem. Thus, we suspect that stable points will be most useful when one of two conditions hold: first, the particular situation involves rapid evolution of technology (a Red Queen scenario), or second, the situation involves interaction between firms, e.g., interconnection, rather than the behavior of a single firm. In this context, we list a few areas that we consider likely fruitful future application of the concept of stable points to adaptive regulation.

1. Universal service Technical innovation may eventually greatly reduce the cost of making broadband available everywhere, including high-cost areas, but today this goal is gated by capital and return on investment. That is, universal service is not a "Red Queen race" where regulation is trying to keep up with rapid change in other parts of the ecosystem. But stable points may still play a role in supporting this goal. Specifically, broadband access implies an interface between the provider and the subscriber, the specification of which determines the nature of the service. Although this specification may change over time as communication technology evolves, this interface could serve as a stable point to allow predictability in the definition of that service - an understanding shared among providers, subscribers and regulators as to how this specification will change over time. Section 3.2 described various approaches, some more predictable than others, to defining broadband. We argue that while the pursuit of universal service may not be driven by rapid innovation, there is still a role for a form of stable point to facilitate effective regulatory intervention.

12 In 2014, the FCC's Technical Advisory Council (a multi-stakeholder group) published a report discussing how to operationalize such "harm claim thresholds", i.e., what level of interference should constitute a harm to the incumbent. http://transition.fcc.govoettac/tacdocs/reports/TACInterferenceLimitsIntrov1.0.pd $\mathrm{f}$ 
2. Copyright In the case of copyright, both rights-holders and those who share infringing content exploit technological innovation as well as legal tools (DMCA), resulting in a race in an ecosystem with rapid technology evolution (for DRM as well as circumvention). However, this Red Queen's Race is about enforcement, not regulation. An open question is whether enforcement issues are similar to regulatory issues, and whether factors that affect rates of change in regulation similarly affect rates of change in approaches to enforcement.

3. Privacy Privacy is a case where regulation (and norms) are clearly struggling to keep up with expanding capabilities and associated complexity created by advances in technology. Privacy debates currently seem centered on the first two requirements in Table 1: agreeing on policy goals and measuring progress toward them. In turn, there are two ways to pose operational goals concerning privacy: restrict what data can be gathered or restrict what can be done with it. But defining some stable points at the information layer may improve the effectiveness of privacy regulation. An example might be the representation and ontology of meta-data that describes different types of data with privacy implications.

We note that the presence of stable points in a system, and a linking of goals and rules to those points, does not change the requirements for effective regulation listed in Table 1. But stable points can slow down the required rate of co-evolution so that rules remain fit for purpose. One must still clearly state goals, measure progress toward the goals, and make judgments about causality. But our hope is that by enabling policy frameworks that are more amenable to adaptation, there will be less pressure for rapid evolution of the frameworks, and sound judgments about issues such as causality will be less problematic.

\subsection{Open questions}

We have oversimplified the frenetic rate of evolution in the technology sector. We acknowledged that in capital-intensive undertakings (like the replacement of our access copper plant with fiber), successful choices in the deployment of capital could give a firm or a technology a stable place in the ecosystem. The Bell system survived for decades through the joint industry-government construction of a regulated monopoly. Once a region of the world has invested to build fiber infrastructure, its vast capacity will survive and serve for an extensive period, as did copper pairs in their day. That fiber, and the firms that own it, will likely become a stable point in the larger ecosystem. One open question is whether society can better shape the character of that stable point during the dynamic period that precedes it or once it emerges.

The IT sector has thus far always been in a state of flux. Might it enter a more stable period, with lower rates of evolution? Moore himself predicted in 2005 that Moore's law would fail in 10 or 20 years (Dubash, 2005), and a more recent prediction in The Economist anticipated a slowing in 2014 (Sutherland, 2013). Some might consider such slowing not such a bad thing (Laird, 2013), some fear it would 
trigger a dramatic economic downturn (Kaku, 2011), and some believe that maturation of the ecosystem will curtail innovation as open, well-specified interfaces are replaced with private, internal and tightly-coupled interfaces (DeVries, 2011). ${ }^{13}$ The linkage between clean interfaces (which produce loosely coupled systems) and innovation is well-understood (Longstaff, 2003) ${ }^{14}$. A slowing of Moore's Law would likely change the pressures for co-evolution between ICT technology and regulation, and reduce pressure on the regulator to evolve rapidly.

The hypothesis of adaptive regulation may be relevant, namely that regulation evolves as one component of a co-evolving complex system. But for the concept to be practically useful, attention must shift from an abstract view to an operational implementation of the theory-a focus on the means and methods that support the clear statement of durable goals, the metrics that are used to deterring progress, and how to design regulatory options that will yield progress. Weak feedback signals may inhibit the ability to measure progress toward specified goals, rendering a possible undesirable outcome of adaptation: fluctuation as the result of battles among ideologies armed with heavy lobbying capital. To confirm or refute this fear, we pose two high-level questions. First, can a society (a country, or any scope of regulation) reach agreement on the desired outcomes for some proposed regulatory intervention, and an agreed means to measure progress toward those outcomes, and second, how much time is required to evaluate its success?

Finally, as we consider how to weave regulation into the design of evolving systems, a challenging question is whether to put stable points into the architecture of cyberspace specifically to facilitate forms of state control and regulation. A conversation around this point would require a combination of technologists and experts in law and the concerns of different governments. The process would be complex, highly technical, highly political, and subtle, with sometimes unanticipated consequences. There are few institutions today where these issues could be discussed in depth, and no definitive way to agree on larger social or state requirements and "throw them over the fence" into the hands of technology architects, since tensions among the interests of governments (both individually and collectively) prevent clear articulation of design requirements. The process of understanding how the state can or should exercise control over the IT sector is likely to proceed in incremental and sometimes fragmentary ways, which is perhaps all we should expect of the real world.

\footnotetext{
13 "Modular technology does not lead inescapably to a modular industry structure. ... The tide will recede slowly, but it is becoming clear that key assets of the web, such as the massive data sets held by Google, eBay, Facebook, and others, have proprietary value and will thus not be made interoperable." (DeVries, 2011, p.106).

14 “'[I]f innovation or localized response to particular problems were a goal, then loosely coupled systems would seem to be in order. ... However, if the goal is standardization across the entire system, then a tight coupling of the entire system (including all subsystems) is more likely to yield the desired outcome." (Longstaff, 2003).
} 


\section{Acknowledgement}

MIT work reported in this paper was supported by the Office of Naval Research under award number N00014-09-1-0597 as part of the DoD Minerva program. UCSD's contribution was supported in part by NSF CNS-1111449 and CNS-1345286. Any opinions, findings, and conclusions or recommendations expressed in this publication are those of the author and do not necessarily reflect the views of the Office of Naval Research or the National Science Foundation.

\section{References}

Belady, L. A., \& Lehman, M. M. (1976). A model of large program development. IBM Systems Journal, 15(3), 225-252.

Boudreau, K., \& Hagiu, A. (2011). Platform rules: Multi-sided platforms as regulators. In Platforms, markets and innovation. Edward Elgar.

Cherry, B. A. (2007). The telecommunications economy and regulation as coevolving complex adaptive systems: Implications for federalism. Federal Communications Law Journal, 59(2).

Cherry, B. A. (2008). Institutional governance for essential industries under complexity: Providing resilience within the rule of law. CommLaw Conspectus: Journal of Communications Law and Policy, 17(1).

Cherry, B. A., \& Bauer, J. M. (2004). Adaptive regulation: contours of a policy model for the internet economy. (Available at http://quello.msu.edu/sites/default/files/pdf/wp-04 -05.pdf)

Clark, D. (1988). The Design Philosophy of the DARPA Internet Protocols. In Sigcomm. ACM.

Clark, D., Chapin, L., Cerf, V., Braden, R., \& Hobby, R. (1991). Towards the future internet architecture. Network Working Group Request for Comments 1287.

Clark, D., \& Landau, S. (2011). Untangling attribution. Harvard National Security Journal, 2.

Clark, D. D. (2009). A multi-theory analysis of long-lived networks. (unpublished working paper, available at http://groups.csail.mit.edu/ana/People/DDC/Longevity.pdf)

Coase, R. H. (1959). The Federal Communications Commission. In (Vol. 2). Journal of Law and Economics. (Available at http://links.jstor.org/sici?sici=00222186\%28195910\%292\%3C1\%3ATFCC\%3E2.0.C0\%3B2-A)

Computer Science and Telecommunications Board. (2002). Broadband: Bringing home the bits. National Academy Press.

Craig, R. K., \& Ruhl, J. B. (2014). Designing Administrative Law for Adaptive Management. Vanderbilt Law Review(1). (http://ssrn.com/abstract=2222009) doi: doi.org/10.2139/ssrn.2222009

DeVries, J. P. (2011). The resilience principles: A framework for new ict governance. JTHTL: Journal on Telecommunications and High Technology Law, 9(1). (Available at http://www.jthtl .org/content/articles/V9I1/JTHTLv9i1 DeVries.PDF) 
Dubash, M. (2005). Moore's Law is dead, says Gordon Moore. (http://news.techworld.com/ operating-systems/3477/moores-law-is-dead-saysgordon-moore/, visited Deceomber 21, 2013)

Federal Communications Commission. (2002). Spectrum Policy Task Force Report, ET Docket No. 02-135.

Federal Communications Commission. (2015). 2015 broadband progress report. (https://www.fcc.gov/reports/2015-broadband-progress-report)

Frischmann, B. M. (2012). Infrastructure: The social value of shared resources. Oxford University Press.

Gawer, A. (2011). Platform dynamics and strategies: From products to services. In Platforms, markets and innovation. Edward Elgar.

Hagiu, A., \& Wright, J. (2011). Multi-sided platforms (Tech. Rep. No. 12-024). Harvard Business School. (available at http://hbswk.hbs.edu/item/6681.html)

Hubbard, R. G., Scott, H., \& Zingales, L. (2008, September). Let's Get the Bank Rescue Right. Wall Street Journal. Retrieved from http://online.wsj.com/news/articles/ SB122221456930869333

ITU-T. (2001). Standards approval. (http://www.itu.int/en/ITU-T/about/Pages/ approval.aspx)

$\mathrm{k}$ claffy. (2011, January). thoughts on ICANNs plans to expand the DNS root zone by orders of magnitude. (http://blog.caida.org/best available data/2011/01/19/thoughts-on -icanns-plans-to-expand-the-dns-root-zone-byorders-of-magnitude/)

Kaku, M. (2011). What happens when computers stop shrinking? (http://www.salon.com/2011/03/19/moores law ends excerpt/, visited December $21,2013)$

kc claffy, \& Clark, D. (2014). Platform models for sustainable Internet regulation. Journal of Information Policy, 4, 463-488.

(http://www.jstor.org/stable/10.5325/jinfopoli.4.2014.0463)

Kirschner, M., \& Gerhart, J. (1998). Evolvability. Proceedings of the National Academy of Science, 95, 8420-8427.

Laird, J. (2013). Why the death of Moore's Law wouldn't be such a bad thing. (http:// www.techradar.com/us/news/computing/why-the-death-of-moore-s-law wouldn-t-be-such-a-bad-thing-1175977, visited December 21, 2013)

Longstaff, P. H. (2003). The puzzle of competition in the communications sector: Can complex systems be regulated or managed? Program on Information Resources Policy. (Available at http:// www.pirp.harvard.edu/pubs pdf/longsta/longsta-p031.pdf)

Manzi, J. (2012). Uncontrolled: The surprising payoff of trial-and-error for business, politics, and society. Basic Books.

McKenzie, H. (2013). Move fast, break things: The sad story of platform, facebook's gigantic missed opportunity. PandoDaily. (http://pando.com/2013/07/23/move- 
fast-break-things -the-sad-story-of-platform-facebooks-gigantic-missedopportunity/, visited December 2013)

Moore, G. E. (1965). Cramming more components onto integrated circuits. Electronics. (reprinted in Proceedings of the IEEE, Vol 86, No 1, January 1998)

Postel, J. (1981). Internet protocol. (Network Working Group Request for Comments 791)

President's Council of Advisors on Science and Technology. (2012). Realizing the full potential of government-held spectrum to spur economic growth.

Ruhl, R. B. (2005). Regulation by adaptive management - is it possible? Minnesota Journal of Law, Science \& Technology, 7(1). (Available at http://mjlst.umn.edu/prod/groups/ahc/ @pub/@ahc/@mjlst/documents/asset/ahc asset 365948.pdf)

Scheuerman, W. E. (2004). Liberal Democracy and the Social Acceleration of Time. Johns Hopkins University Press.

Schilling, M. A. (2011). Protecting or diffusing a technology platform: Tradeoffs in appropriability, network externalities, and architectural control. In Platforms, markets and innovation. Edward Elgar.

Sutherland, B. (2013). No Moore? (http://www.economist.com/news/21589080 -golden-rule-microchips-appears-be-coming-end-no-moore, visited Decem- ber 21, 2013)

van Schewick, Barbara. (2010). Internet Architecture and Innovation. MIT Press. VanValen, L. (1973). A new evolutionary law. Evolutionary Theory, 1, 1-30.

Whitt, R. S. (2009). Evolving broadband policy taking adaptive stances to foster optimal internet platforms. CommLaw Conspectus: Journal of Communications Law and Policy, 17(2). 